\title{
UN ENFOQUE OPERATIVO DE LA PLANIFICACIÓN DEL DESARROLLO
}

\author{
AlBert WATERSTON *
}

\section{El ENFOQUe CONVENCIONAL DE LA PLANIFICACión}

\section{La situación del desarrollo planificado}

Después de que en los dos últimos decenios la planificación prácticamente logró la aceptación mundial, ahora está comenzando a ponerse en tela de juicio su utilidad como instrumento para acelerar el ritmo del desarrollo. Es posible que, en parte, se trate de una reacción contra el entusiasmo excesivo de los que esperaban que la planificación conduciría automáticamente a un desarrollo rápido. Pero hasta los menos ingenuos se sienten desalentados con los resultados del desarrollo planificado.

Ante la actitud general de desilusión, en el número de CERES, publicación de la FAO, de mayo-junio de 1968, Hans Singer lamenta que con frecuencia se califique de "decenio de la frustración" al Decenio del Desarrollo de los años 60. Si bien es verdad que ese calificativo es "algo exagerado", no deja de haber justificación para utilizarlo. Es un hecho que, aun tomando en cuenta las reservas respecto a la confiabilidad estadística de los datos relativos a los países pobres, las cifras disponibles señalan que a pesar de la dependencia cada vez mayor en la planificación a partir de la Guerra, la tasa anual de crecimiento del producto interno bruto (PIB) en todos los países menos desarrollados no ha experimentado, prácticamente, ninguna mejoría desde el decenio de $1950 .{ }^{1}$ Esto es lo que ha sucedido en el caso de Africa. ${ }^{2}$ Ahora bien, en América Latina, y especialmente en el Asia meridional y sudoriental, la tasa media anual de crecimiento del PIB ha disminuido. ${ }^{3} \mathrm{La}$ situación

* Conferenciante del Instituto de Desarrollo Económico del Banco Mundial. Este trabajo refleja mis puntos de vista, y no los del Instituto ni los del Banco. Me complace expresar mi reconocimiento a los señores Loreto $M$. Domínguez P., David Henderson, Raj Krishna y Eugene R. Schlesinger por sus valiosas sugerencias.

1 Según el World Economic Survey, 1967, Parte primera (p. 17), de las Naciones Unidas, las tasas medias de crecimiento del PIB descendieron del $4.6 \%$ en 1955-60 al $4.4 \%$ en 1960-65, y del $2.3 \%$ per capita al 1.9\%; según el Informe Anual de 1968 del Banco Mundial (p. 51), las tasas medias de crecimiento del PIB subieron del $4.7 \%$ en $1950-60$ al $4.8 \%$ en 1960-66, pero bajaron del $2.4 \%$ per capita al 2.3 .

2 Según las Naciones Unidas (ibid.), las tasas de crecimiento del PIB en Africa aumentaron del $4.3 \%$ en $1955-60$ al 4.5 en $1960-65$, y del $2.1 \%$ per capita al 2.3; según datos del Banco Mundial, las tasas de crecimiento del PIB en Africa fueron, como promedio, del $4.0 \%$ en 1950-60 y 1960-67, pero el PIB per capita declinó ligeramente, del $1.7 \%$ en $1950-60$ al 1.6 en $1960-67$.

3 Según las Naciones Unidas (ibid.), las tasas de crecimiento del PIB en la América Latina descendieron del $4.6 \%$ en $1955-60$ al 4.4 en $1960-65$, y del $1.9 \%$ 
difícil en que se encuentran los países menos desarrollados puede también inferirse de su participación descendente en el ingreso mundial. En 1950, el $60 \%$ más pobre de la población del mundo recibió el $13.2 \%$ de dicho ingreso, y en 1964 su participación fue sólö del $11.1 \% .^{4}$

Al renovar o intensificar sus ataques contra el concepto de planificación del desarrollo en general, los que se oponen a la intervención gubernamental en la economía no han dejado de señalar cuán poco satisfactorio ha sido su historial. ${ }^{5}$ A esto responden los defensores del sistema aceptado de planificación que el problema no estriba en el enfoque convencional de la planificación, sino en el hecho de que los gobiernos no observan los preceptos y normas establecidos por los planificadores. ${ }^{6}$ En realidad alegan que si los hechos no concuerdan con la teoría, tanto peor para los hechos. No debe considerarse que esta actitud es arrogante, ya que lo que quieren decir es que los gobiernos, a través de sus decisiones o de su inacción, crean o perpetúan situaciones que son desfavorables para la planificación del desarrollo.

En efecto, es posible que el quid del problema radique en la acción inadecuada o en la negligencia del gobierno, péro la importancia de esos elementos difiere de la que le atribuyen los defensores del enfoque tradicional a la planificación. Es decir, que si debido a la naturaleza de la planificación convencional o al medio ambiente de los países de bajos ingresos no resulta realista esperar que en dichos países existan las condiciones necesarias para el funcionamiento satisfactorio de la planificación convencional, tal vez sea más sensato tratar, por lo menos al principio, de modificar la teoría más bien que los hechos, o sea, la planificación convencional en lugar de la actuación de los dirigentes políticos. En ese caso, cabe preguntarse cómo ha de llevarse a cabo dicha modificación. Ése es el tema que se abordará en el presente trabajo.

\section{La planificación convencional}

En su libro sobre la planificación del desarrollo, el profesor W. Arthur Lewis aconseja a los países que planifican su desarrollo económico la adopción de tres clases de planes: "Debe haber un plan

per capita al 1.7; según et Banco Mundial (ibid.), las tasas de crecimiento del PIB bajaron del $4.9 \%$ en $1950-60$ al $4.7 \%$ en 1960-66, y del $1.9 \%$ per capita al 1.7 .

Según las Naciones Unidas (ibid.), las tasas de crecimiento del PIB en el Asia meridional y sudoriental disminuyeron del $4.2 \%$ en $1955-60$ al 3.5 en $1960-65$, y del $2.0 \%$ per capita al 1.2 ; según el Banco Mundial (ibid.), las tásas de crecimiento del PIB en el Asia meridional descendieron del 3.6\% en 1950-60 al 3.4 en 1960-66, y del $1.7 \%$ per capita al 0.5 .

4 Véase James $\mathrm{H}$. Weaver y Leroy Jones, "International Distribution of Income: 1950-1964", Journal of Economic Issues (de próxima aparición).

5 Véase, por ejemplo, P. T. Bauer, "Dissent on Development", Scottish Journal of Political Economy, Vol. XVI, Núm. 1, febrero, 1969, Universidad de Glasgow, Gran Bretaña, pp. 75-94; Gerald Sirkin, The Visible Hand: The Fundamentals of Economic Planning, Nueva York, McGraw-Hill Book Company, 1968; y John Jewkes, The New Ordeal by Planning: The Experience of the Forties and Sixties, Londres, Macmillan, 1968.

6 No se trata de personal de segundo orden, sino de verdadero valer. Basta visitar las oficinas centrales de planificación en casi cualquier parte del mundo menos desarrollado para escuchar esa réplica. 
anual, un plan a plazo medio y un plan de perspectiva a largo plazo".7 Jan Tinbergen, en su obra sobre la planificación del desarrollo, también recomienda que los países en desarrollo preparen planes a plazo largo y medio, así como planes anuales. ${ }^{8}$ Quizá sería exagerado sugerir que hay un consenso entre los especialistas en planificación acerca de la manera exacta de abordar la planeación del desarrollo. Pero basándose en lo que se ha escrito sobre ese tema, es razonable manifestar que casi todos los especialistas coinciden en que el desarrollo nacional exige la preparación de las tres clases de planes que Lewis y Tinbergen recomiendan.

Al igual que la mayoría de los que escriben sobre la planificación del desarrollo, estos autores sugieren que el proceso debe iniciarse con un plan a largo plazo que refleje una evaluación de las perspectivas de desarrollo de un país en un período de quince a veinte años. El desarrollo requiere tiempo, y por esa razón, arguyen ellos, la formulación de metas a largo plazo es fundamental para asegurar que las decisiones en materia de desarrollo sean compatibles con los objetivos, necesidades y posibilidades a largo plazo. Se recomienda a los planificadores la preparación de planes de largo alcance por considerar que constituyen un instrumento adecuado para el estudio sistemático de los costos futuros y otras repercusiones de las decisiones en materia de inversión. También se recomiendan porque proporcionan orientación a los planificadores y demás personal encargado de la adopción de esas decisiones, al indicarles los atascamientos que se producirán según la economía vaya creciendo, a menos que se tomen medidas preventivas con la antelación necesaria. ${ }^{9}$

Los especialistas en planificación recomiendan asimismo que, una vez preparado un plan a largo plazo, se proceda a la formulación de uno a plazo medio. Éste suele abarcar de tres a siete años, pero puede extenderse hasta diez años. Por ejemplo, Tailandia ha tenido un plan de seis años; Irán, uno de siete años; Indonesia, uno de ocho años; Liberia, uno de nueve años, y Chile, uno de diez años. No obstante, el plan quinquenal es el que goza de más popularidad. Los planes a plazo medio son más detallados que los planes a largo plazo, o de perspectiva. Son los planes a los que dedican los planificadores, por lo general, la mayor parte de su tiempo. Su preparación puede tomar entre dieciocho meses y tres, cuatro y aun cinco años.

Por último, existe el plan anual. La naturaleza y la orientación de un-plan de esta índole se determinan en función del plan a plazo medio, y las de este último están regidas por el plan de largo alcance. El plan anual es esencial para una buena planificación porque proporciona los medios necesarios para el funcionamiento de los planes a plazo mediano. En realidad, en los países socializados, que necesitan los planes anuales tanto como los países de economía mixta planificada, éstos se conocen como planes operacionales.

Puesto que es un hecho que casi toda la literatura sobre la planificación del desarrollo, así como la mayor parte del tiempo e interés de

7 Development Planning. The Essentials of Economic Policy, Nueva York, Harper and Row, 1966, p. 150. (Hay traducción del F. C. E., México, 1968.)

8 Development Planning, Londres, World University Library, 1967, pp. 52-53.

9 Lewis, op. cit., p. 150. 
los planificadores, se concentra en los planes a plazo medio, conviene examinar brevemente los procedimientos que los especialistas consideran esenciales para la formulación de un buen plan de esa índole. Los técnicos concuerdan, en general, en que la preparación de un plan global a plazo medio (global en el sentido de que abarca tanto el sector privado como el público) entraña el empleo de dos procedimientos de aplicación simultánea. Uno de éstos procede de lo general a lo particular, y se denomina a veces "planificación desde arriba", en tanto que el segundo va de lo particular a lo general, y se conoce como "planificación desde abajo".

La "planificación desde arriba" comienza con la fijación por las autoridades políticas de objetivos de desarrollo claramente definidos, seguida de la formulación por el organismo central de planificación de un plan global o agregado que responda a dichos objetivos. Se procede a través de la elaboración de una serie de proyecciones relacionadas entre sí, cuyas hipótesis constituyen un modelo de crecimiento para el plan proyectado.

Por lo general, mediante un modelo se calcula el efecto de una tasa supuesta de crecimiento sobre el consumo, el ahorro y la inversión tanto del sector público como del privado, sobre las importaciones y las exportaciones, y sobre el nivel de empleo, así como las repercusiones para la demanda y la oferta que supone el logro del producto o ingreso nacional supuestos. Se llevan a cabo diversos cálculos con el fin de relacionar los insumos de mano de obra, materias primas, tierra y capital con los productos resultantes. También se efectúan otros cálculos sobre las relaciones entre el ingreso que ha de generarse y gastarse en los sectores público y privado para fines de consumo e inversión, de importaciones y exportaciones, etc. Estos cálculos pueden abarcar desde sencillas estimaciones empíricas o meras conjeturas, hasta complejas formulaciones que comprendan la utilización de tablas de insumo-producto, la programación lineal o incluso técnicas econométricas más refinadas. Los resultados se someten a pruebas para determinar si están en consonancia con las metas globales, si son compatibles entré sí, y si se encuentran dentro de los límites de los recursos disponibles. ${ }^{10}$

Una vez preparado y aprobado el modelo agregado, el procedimiento de "planificación desde arriba" exige que se dividan o "desagreguen" las metas globales en programas sectoriales relacionados entre sí y, a veces, en planes regionales. En los casos en que el plan global se divide en planes regionales, éstos a su vez deben subdividirse en programas sectoriales. Cada programa sectorial, sea nacional o regional, tiene sus propias metas globales de crecimiento, inversión, insumos y producción. En la última fase del procedimiento de "planificación desde arriba", se indica la medida en que los diferentes sectores económicos y sociales, sean éstos nacionales o regionales, requieren capacidad adicional para alcanzar las metas del plan y, en ciertas ocasiones, se enumeran los proyectos necesarios para proporcionar esta capacidad.

Al mismo tiempo que se lleva a cabo la "planificación desde arriba", ha de procederse también a la "planificación desde abajo", la que

10 Albert Waterston, Planificación del desarrollo: lecciones de la experiencia, México, Fondo de Cultura Económica, 1969, pp. 70-71. 
incluso debe iniciarse antes que aquélla debido a que toma más tiempo. En el sector público, esto supone que cada ministerio, departamento y empresa pública debe enumerar todos los proyectos que espere 1levar a cabo en su totalidad o en parte durante el período del plan, los clasifique de acuerdo con su prioridad, y los combine en programas sectoriales o subsectoriales coherentes, con base en objetivos claramente definidos. Estos programas deben presentarse en una fecha determinada al organismo central de planificación, para que éste los combine, y los coordine entre sí y con los recursos disponibles.

Mientras que los ministerios, departamentos y organismos preparan sus programas, el organismo central de planificación obtiene información de los inversionistas de los sectores industrial, agrícola, minero y otros, o de las asociaciones de intereses manufactureros, agrícolas, mineros y de otra índole, con el fin de determinar qué planes tienen ( $o$, en el caso de las asociaciones, sus miembros) en lo que se refiere a la ejecución de nuevos proyectos o la ampliación de las instalaciones existentes. El organismo central de planificación puede confeccionar una lista que comprenda, por lo menos, los proyectos más importantes que los inversionistas privados se propongan iniciar, proseguir o llevar a cabo durante el período del plan; y hacer un cálculo del nivel probable de la inversión privada en los sectores industrial, agrícola, minero y otros durante dicho período. Estos totales parciales de los sectores se suman para obtener el total correspondiente a la inversión privada, el que, al añadirse al total propuesto por los organismos gubernamentales para su inversión en el sector público, viene a representar un plan general de inversión.

Si el plan nacional de desarrollo comprende planes regionales, en cada uno de ellos los proyectos de los sectores público y privado deben organizarse por sectores económicos y sociales, y posteriormente es necesario que se sumen las cifras de todas las regiones para determinar la inversión total.

Los totales parciales por sector y por región, así como los agregados obtenidos mediante el procedimiento de "planificación desde abajo", probablemente no coincidirán con los totales correspondientes derivados del proceso de "planificación desde arriba". Los dos grupos de cálculos deben considerarse como "primeras aproximaciones" que habrán de conciliarse posteriormente. En ese proceso, es posible que sea necesario reducir algunas cifras del modelo original y aumentar otras. A su vez, esas modificaciones exigirán la revisión de otras cifras, así como la implantación de cambios en los supuestos y metas. Mediante aumentos y disminuciones y la utilización de un sistema empírico basado en un proceso que produce una serie de llamadas "aproximaciones sucesivas", los dos grupos de cálculos preparados a través de los procedimientos de "planificación desde arriba" y "planificación desde abajo" son "empujados", "aguijoneados" y "podados" paulatinamente para lograr una mayor proximidad aritmética, hasta que surja un plan global a plazo medio integrado, dotado de coherencia interna y dividido por sectores, que con frecuencia comprende entre sus elementos una lista de proyectos y, en ocasiones, planes regionales.

La planificación convencional tiene grandes virtudes. En primer 
lugar, proporciona la perspectiva necesaria para evaluar las decisiones en materia de inversión en función de los costos futuros y los objetivos de desarrollo a largo plazo. En segundo lugar, gracias a la visión de conjunto que ofrece de la economía, hace posible evitar la distribución inadecuada de los escasos recursos disponibles, ya que brinda la oportunidad de establecer una comparación entre las necesidades de los sectores público y privado al permitir la determinación de las oportunidades marginales que prometan dar los resultados apetecidos al más bajo costo. En tercer lugar, gracias a su coherencia interna, ofrece cierta protección contra la aparición de atascamientos y desequilibrios inesperados que puedan impedir el crecimiento. Por último, los procedimientos seguidos en la preparación de los planes a plazo largo y medio y anuales contribuyen a familiarizar a los participantes con los elementos de la planificación del desarrollo.

\section{Defectos metodológicos}

No obstante, es preciso tener presente que en la preparación de planes en países de bajos ingresos los planificadores utilizan instrumentos que, en gran medida, han sido confeccionados en los países más adelantados y con miras a su utilización en esos países. La experiencia suscita ciertas dudas respecto a la utilidad de esta "transferencia de tecnología". Por ejemplo, la aplicación en países menos desarrollados, en que la economía de subsistencia desempeña un papel importante, de los conceptos, categorías y políticas de precios utilizados en un sistema de cuentas nacionales elaborado para países industrializados puede dar lugar a conclusiones erróneas o inexactas. Debido a que la distribución del ingreso es muy desigual, en los países pobres el ingreso per capita es un dato mucho menos significativo que en los más adelantados, en que los ingresos están distribuidos de manera más igualitaria. El incremento del ingreso per capita también constituye un elemento menos eficiente para medir el progreso en los países pobres que en los más desarrollados, ya que no toma en cuenta los cambios institucionales que son indispensables para salir de la etapa del subdesarrollo. Tampoco hay pruebas de que el empleo de tiempo, dinero y esfuerzos en la preparación de enormes cuadros de insumoproducto constituya para los países de bajos ingresos una inversión de recursos financieros y técnicos escasos que sea siquiera remotamente tan útil como en el caso de las naciones más adelantadas. Y es dudoso que los modelos de planificación utilizados para elaborar planes de desarrollo a plazo medio en los países de bajos ingresos hayan contribuido mucho a su desarrollo.

En los últimos años se han logrado avances importantes en lo que se refiere a las técnicas de planificación. Ahora bien, la mayor parte de los modelos que se utilizan realmente en los países de bajos ingresos en relación con los planes a mediano plazo suponen una simplificación tan excesiva de la realidad que a menudo proporcionan ideas erróneas. Como ha señalado Paul Streeten, es posible que los resultados sean elegantes y atractivos desde un punto de vista técnico, pero carecen de órganos vitales. Por ejemplo, a pesar de que la esencia del desarrollo es el dinamismo, los modelos a menudo parten del supuesto 
de parámetros estables (por ejemplo, coeficientes incrementales de capital-producto). Al propio tiempo, es posible que los modelos sean demasiado "dinámicos", en el sentido de que las proyecciones para cinco, diez o más años produzcan resultados de dudosa exactitud, dado el estado actual del arte de la planificación. Casi siempre se supone la existencia de relaciones lineales entre las variables (en forma de coeficientes constantes de producto-insumo), a pesar de que en la práctica esas relaciones no son comunes, mientras que su ausencia sí lo es. Los modelos suelen describir las economías de los países de bajos ingresos en función de un agregado para cada uno de los elementos, tales como el consumo, el ahorro, la inversión, la producción, el ingreso per capita, etc., mientras que la naturaleza dual o triple de la mayoría de los países de bajos ingresos hace que se necesiten dos o tres valores para cada uno. A menudo abarcan solamente dos sectores (por ejemplo, la agricultura y la industria, o los bienes de producción y de consumo), en lugar del mayor número de sectores importantes que suele haber hasta en economías sencillas. Por lo general, atribuyen el desarrollo a un solo factor, el capital, si bien se reconoce que los factores políticos, sociales e institucionales afectan, en forma decisiva, los resultados que produce la inversión. Y los cálculos de los modelos se efectúan a base de precios constantes, a pesar de que los cambios en los precios con frecuencia dan lugar a distorsiones en las relaciones entre sectores y a cambios en las necesidades de financiamiento para el plan.11

\section{La insuficiencia de datos}

No obstante, la elaboración de un modelo que refleje en forma adecuada aun la economía de un país pequeño de bajos ingresos y las fuerzas más importantes que determinan su desarrollo, exigiría, por lo general, el empleo de técnicas mucho más complicadas que las aplicables, en la práctica, en la mayor parte de los países de bajos ingresos. ${ }^{12}$ Por otra parte, se sentiría en forma mucho más pronunciada la insufi. ciencia de datos que dificulta la planificación, aun en el caso de los modelos más sencillos. Todo el que ha trabajado en ese campo sabe que, por lo general, en los países de bajos ingresos los datos estadís ticos no son confiables o sencillamente no existen. No es sólo que las relaciones incrementales de capital-producto a menudo sean poco más que conjeturas, sino también que los datos básicos relativos a la población, el crecimiento demográfico, la producción y el ingreso nacional, así como muchos de los componentes de este último, o bien son de dudosa exactitud o no existen.

Debido a la insuficiencia de datos, resulta difícil la planificación, tanto desde un punto de vista cualitativo como cuantitativo, pero el

$11 \mathrm{Ha}$ sido Enrique Lerdau quien me ha señalado este punto. En ocasiones se considera que el aumento porcentual de los precios constituye un factor correctivo, pero, aparte de la dificultad de prever los aumentos de los precios, no existe manera práctica de pronosticar las distorsiones sectoriales a que podrían dar lugar los cambios en los precios.

12 Alec Nove habla de un destacado economista que inició una conferencia con estas palabras: "Partiré del supuesto de competencia perfecta, pues, de lo contrario, las matemáticas resultarán demasiado difíciles". 
peligro de que se multipliquen los errores es grande cuando se incorporan datos inexactos en un plan que, por su propia naturaleza, ha de estar dotado de coherencia interna. En muchos planes, solamente hay coherencia en lo que se refiere a la deficiencia de las estadísticas, y no a los hechos..$^{13}$ Lo peor es que, si bien un planificador cuidadoso conoce las limitaciones de sus datos, en su mayor parte los dirigentes políticos no tienen conciencia de la poca confiabilidad de los datos que han de utilizar los planificadores. En consecuencia, suelen aceptar sin grandes reservas las cifras básicas contenidas en los planes que adoptan, concediendo así reconocimiento oficial al error y a la labor incompleta.

5. La inestabilidad, la incertidumbre, la voluntad de alcanzar el desarrollo y los problemas administrativos

A pesar de su gravedad, las deficiencias en la metodología y en los datos no son las razones más importantes de que el desarrollo planificado no haya estado a la altura de las previsiones ni de las necesidades. La experiencia demuestra que, en lo que respecta a sus planes de desarrollo, son pocos los países de bajos ingresos que poseen los elementos necesarios para la ejecución de planes a plazo mediano, tales como 1) estabilidad política, 2) seguridad económica, 3) voluntad política y 4) capacidad administrativa necesaria. La planificación convencional parte del supuesto de que el cambio, aunque inevitable, ocurre en un ambiente generalmente estable. Pero es un hecho que el cambio - súbito, con frecuencia imprevisible y de gran alcance- en la situación política y económica de los países, sobre todo los menos desarrollados, ha pasado a ser una característica del mundo moderno.

\section{a) La inestabilidad politica}

Los acontecimientos de los últimos diez años ponen claramente de manifiesto que la inestabilidad política es la norma en los países de bajos ingresos. Durante ese período, ha habido por lo menos 56 golpes de estado en los países menos desarrollados. ${ }^{14}$ Las repercusiones de estos cambios sobre la planificación pueden apreciarse mejor si recordamos que los gobiernos rara vez siguen adelante con los planes de desarrollo a plazo medio o largo de sus predecesores. $\mathrm{Y}$ aun cuando no haya cambios en los niveles superiores de un gobierno, la inestabilidad política puede impedir la ejecución de tales planes. Por ejemplo, ésa era la situación en el Perú antes del golpe militar de 1968, ya que hubo seis gabinetes durante los cinco años que el presidente Belaúnde Terry estuvo en el poder; y lo mismo sucede en Nigeria, donde la contienda civil alcanza proporciones que entorpecen el funcionamiento ordenado del gobierno.

13 A pesar de que las comprobaciones de coherencia pueden llevar al descubrimiento de que las estimaciones carecen de ella, este descubrimiento no conlleva, por necesidad, la capacidad de rectificar la situación. Si los datos son erróneos, la modificación por los planificadores de las cifras para darles coherencia puede incrementar aún más los errores.

14 The Economist, 1: de marzo de 1969, p. 601. 


\section{b) La incertidumbre económica}

La excesiva incertidumbre económica afecta también la preparación y ejecución de planes de desarrollo en los países de bajos ingresos. En países como Brasil, Chile, Ghana, Indonesia y Sierra Leona, entre otros, en que la inflación o la estabilización de la balanza de pagos es un motivo de preocupación, los dirigentes políticos y las autoridades conceden invariablemente mayor prioridad a los problemas más inmediatos que a los planes de desarrollo a plazo largo. El descenso inesperado del monto de la ayuda y los préstamos extranjeros e internacionales también han dificultado en algunos países la ejecución de planes a plazo medio. Debido a considerables fluctuaciones de los precios de los productos primarios de exportación, los ingresos en divisas a veces son inferiores al nivel previsto, razón por la cual a algunos países les resulta difícil o imposible alcanzar las metas de inversión establecidas.

\section{c) La voluntad politica de alcianzar el desarrollo}

La aceptación más bien formalista de la planificación del desarrollo por los dirigentes políticos de algunos países de bajos ingresos constituye otro obstáculo para la ejecución de los planes globales a plazo medio. El deseo de muchos dirigentes políticos de retener el control de las decisiones en materia de inversión y de poder improvisar se manifiesta cuando llega el momento de poner en ejecución los planes de desarrollo. Esta actitud de los dirigentes políticos existe también en países con gobiernos de coalición o cuyos ministerios técnicos y financieros gozan de gran poder.

\section{d) El factor administrativo}

Por último, es preciso tener en cuenta que la planificación convencional parte del supuesto de que se contará con una estructura administrativa satisfactoria y con personal especializado, elementos ambos que no existen en la mayoría de los países de bajos ingresos. En su mayor parte, a los países pobres les resulta imposible emplear los procedimientos de formulación de las tres clases de planes que son indispensables para la planificación convencional. En los casos en que se preparan planes a largo plazo, suelen ser rudimentarios y, por lo general, existen apenas en forma inédita. Los países que formulan planes anuales con regularidad son la excepción y no la regla. Asimismo, son pocos los países en que los planificadores han logrado preparar un plan a plazo medio aplicando tanto el procedimiento de "planificación desde arriba" como el de "planificación desde abajo". En la mayoría de los casos, los planes a plazo medio se preparan mediante la aplicación del primero y no del segundo, debido a que la información sobre proyectos y sectores que es preciso obtener de los ministerios, departamentos y organismos técnicos para la "planificación desde abajo" o bien no existe o es tan deficiente que resulta, en gran medida, inaceptable para una planificación realista. En definitiva, la mayor parte de los planes en los países de bajos ingresos se pre- 
paran casi exclusivamente mediante el procedimiento de "planificación desde arriba", porque el organismo central de planificación puede establecer cálculos para suplir la falta de datos.

Cuando llega el momento de poner en práctica sus planes de desarrollo, son pocos los gobiernos de países menos desarrollados que cuenten con un mecanismo administrativo capaz de mantener la disciplina necesaria para la ejecución de planes a plazo medio.

\section{La planificación en la práctica}

La inestabilidad política, la incertidumbre económica, la aceptación formalista de los planes y los problemas administrativos son factores todos que explican, en gran parte, por qué ha sido posible que descendieran las tasas de crecimiento de países con poca experiencia en materia de planificación, mientras mejoraban las técnicas respectivas. Por consiguiente, no es irrazonable preguntarse si el método convencional de planificación, tal como se aplica a estos países, no debería ser objeto de una revisión.

En realidad, debido a la inestabilidad y la incertidumbre de los últimos años, lo mismo podría decirse respecto de países que tienen considerable experiencia en actividades de planificación. Por ejemplo, países con bastante práctica en la planeación del desarrollo han tenido que abandonar sus planes a plazo medio, al resultarles imposible resolver los problemas dentro del marco de los mismos debido a circunstancias imprevistas. Tras dos guerras y dos períodos de sequía en tres años, la India tuvo que postergar su Cuarto Plan Quinquenal de 1965-1969 y recurrir, entretanto, a planes anuales. Por considerar que el ritmo de su economía se estaba acelerando demasiado, el Japón primero abandonó su plan decenal de 1961-1970, y posteriormente el plan quinquenal de 1964-1968 con que lo había sustituido. A pesar de que las directrices para el plan quinquenal de la URSS de 1966-1970 fueron aprobadas en 1966, dicho plan nunca llegó a adoptarse; en cambio, en diciembre de cada año se anuncian metas para el año siguiente. Aunque no se han dado a conocer las razones a que obedece esa actuación, es posible que se deba, por lo menos en parte, a que el nuevo sistema parcialmente descentralizado de ejecución del plan dio lugar a elementos de incertidumbre que no pueden abordarse con eficacia dentro del marco del plan quinquenal. Cuando el gobierno yugoslavo presentó al Parlamento su plan septenal de 1964-1970, éste rehusó aprobarlo durante dos años, formulándose en vez planes anuales. (En último término, se aprobó en forma de plan quinquenal para 19661970.) En Francia, no hubo otra alternativa que descartar el plan quinquenal de 1966-1970, ya que como consecuencia de los disturbios civiles de mayo de 1968 el gobierno tuvo que autorizar un incremento de los sueldos y los salarios que afectó tanto el logro de las metas del plan, que no resultó práctico revisarlo.

\section{a) La planificación aparente}

Dadas las circunstancias, no es extraño que en los países de bajos ingresos los planes nacionales de desarrollo a plazo medio, bien sean 
adecuados o deficientes, vengan a ser, en mayor o menor grado, instrumentos que los ministerios, departamentos u organismos técnicos encargados de la ejecución de los planes no toman en cuenta o eluden. En la práctica, estos organismos siguen, por lo general, un sistema "por proyectos", y muchos de esos proyectos no guardan relación con los planes nacionales de desarrollo ni tampoco entre sí. Los ministerios, departamentos y organismos técnicos de la mayor parte de los países carecen de personal competente para a) determinar, evaluar y preparar buenos proyectos, $b$ ) establecer el orden de prioridad de los proyectos con arreglo a criterios económicos generales, c) ejecutar los proyectos de acuerdo con un programa cronológico satisfactorio y d) encargarse del funcionamiento de los proyectos después de terminados. En general, hay escasez de proyectos debidamente preparados y listos para iniciarse, y es difícil encontrar programas coherentes para los sectores económicos y sociales básicos. La ausencia de proyectos reduce las oportunidades de realizar inversiones productivas. A menudo se aprueban e inician proyectos que probablemente producirán un rendimiento bajo con relación a los costos, por ser los únicos disponibles. La falta de alternativas en materia de inversión hace que los criterios económicos para las inversiones, las técnicas de los precios de cuenta y las estimaciones de la rentabilidad no sean más que una ficción, ya que a menudo no tiene objeto su aplicación al único proyecto de un sector determinado que está listo para iniciarse. La escasez de alternativas en materia de inversión favorece a los que prefieren seleccionar proyectos de valor dudoso, ya que pueden alegar que si se rechaza el proyecto que patrocinan, no se encontrará un sustituto adecuado.

\section{b) La planificación verdadera}

En la mayoría de los países de bajos ingresos, el que determina en definitiva qué proyectos son aceptados por el gobierno es el organismo presupuestario, que con frecuencia no atiende las recomendaciones del organismo central de planificación o del plan nacional de desarrollo. Por esta razón, en muchos países el presupuesto gubernamental se aproxima más a constituir un plan de inversiones públicas que el plan nacional de desarrollo. Ahora bien, como en la mayor parte de los países de bajos ingresos el presupuesto contiene una exposición incompleta de las inversiones públicas, y, por otra parte, su organización y administración son deficientes, ya que utiliza sistemas y procedimientos concebidos con miras al logro de los objetivos limitados de una era pasada más bien estacionaria, no suele ser un instrumento adecuado para satisfacer las necesidades más dinámicas del desarrollo planificado en las circunstancias actuales.

Debido a que los ministerios y departamentos técnicos a menudo no pueden suministrar cálculos fidedignos de los costos y beneficios de los proyectos, ni tampoco estimaciones seguras del período de construcción, las autoridades presupuestarias con frecuencia hacen reducciones "generales" en las solicitudes de asignaciones, en lugar de eliminar los proyectos de baja prioridad. Debido al empleo de las llamadas cuentas "varias" o "transitorias", en que se agrupan indiscri- 
minadamente los gastos correspondientes a diferentes proyectos y programas, y a los retrasos en el cierre de estas y otras cuentas, la información respecto a los gastos pasados para proyectos y programas es inadecuada. Esto, a su vez, da lugar a que las asignaciones presupuestarias para proyectos y programas se determinen sin tomar en cuenta la experiencia anterior.

Hay una diferencia considerable entre la teoría y la práctica en la movilización y distribución de los recursos públicos, el control de los gastos y la estimación del efecto de la inversión pública sobre los gastos ordinarios. La utilización de sistemas arcaicos de clasificación presupuestaria hace que resulte difícil o imposible establecer una relación entre los presupuestos de capital y los ordinarios, o entre los presupuestos y los planes de desarrollo, así como estimar el efecto de aquéllos sobre el desarrollo. Es un fenómeno común que algunos ministerios incurran en gastos excesivos y que lo contrario suceda en otros, y con frecuencia no hay ningún funcionario que conozca el volumen de las inversiones en el sector público, ni si el monto es superior al total previsto de recursos financieros.

Casi sin excepción, los planes a plazo medio no son complementados por planes anuales, indispensables para su funcionamiento satisfactorio. En cambio, es común que el organismo central de planificación prepare un presupuesto público parcial "de desarrollo", que a menudo comprende partidas no relacionadas con el desarrollo, tales como la construcción de prisiones, manicomios o edificios para las embajadas en el exterior, al tiempo que excluye partidas de desarrollo, tales como la investigación agrícola y la capacitación de personal para la administración de los proyectos de desarrollo. Por el contrario, los gastos de investigación y capacitación, a pesar de que a menudo están relacionados con los proyectos incluidos en el "presupuesto de desarrollo" y con frecuencia son tan importantes como las inversiones de capital para fines de desarrollo, suelen estar incluidos en el presupuesto ordinario, que generalmente es preparado por el organismo presupuestario. Puesto que en los países de bajos ingresos la coordinación entre el organismo central de planificación y el organismo presupuestario suele ser deficiente, con frecuencia no se hace provisión de fondos para atender la conservación y funcionamiento de los proyectos terminados. Esto explica, en parte, la existencia de escuelas sin maestros; de hospitales sin equipo, médicos ni enfermeras; y de caminos nuevos que se deterioran con rapidez, en ciertas ocasiones antes de quedar terminados.

\section{c) Planificiación de la asistencia técnica}

En relación con la prestación de asistencia técnica a los países de bajos ingresos, los países y organismos internacionales donantes se basan, por lo general, en los planes nacionales de desarrollo como puntos de referencia. Puesto que, en la práctica, con frecuencia se hace caso omiso de los planes, es posible que los programas de asistencia técnica encaminados a coadyuvar a su preparación apenas se relacionen con las actividades de inversión pública que se estén llevando a cabo. Esto no sucede en el caso de la asistencia técnica que las entidades extran- 
jeras e internacionales proporcionan para los proyectos en que están interesadas. Ahora bien, estos proyectos constituyen solamente una pequeña proporción de la inversión pública total en los países en desarrollo. Sin embargo, pese a la gran falta de experiencia en materia de proyectos en los países de bajos ingresos, la mayoría de los proyectos que en ellos se financian con recursos internos carecen de asistencia técnica. Lo mismo sucede en lo que respecta al mejoramiento de los presupuestos. Puesto que la capacidad de un gobierno para determinar, preparar y ejecutar proyectos productivos, así como para ejercer un control presupuestario adecuado sobre los gastos, constituye un factor determinante básico de los rendimientos de la inversión pública, es comprensible que a menudo no se alcancen las metas de dicha inversión.

\section{La PLANIFICACIÓN DESDE OTRO PUNTO DE VISTA}

\section{El mejoramiento de los modelos macroeconómicos}

De lo anteriormente expuesto, se desprende claramente que el sistema aceptado de planificación tiene, a la vez, defectos y virtudes. Lo importante es eliminar los defectos al propio tiempo que se retienen las virtudes. En parte, ese objetivo puede lograrse mediante una mejor formulación de los planes. Por ejemplo, los modelos macroeconómicos de planificación pueden ser más realistas si se convierten los parámetros en variables, es decir, si se incluyen en los modelos de planificación factores importantes que suelen considerarse que caen fuera del ámbito de los modelos. A este respecto, cabe considerar los factores administrativos y políticos que, por lo general, se excluyen de la mayoría de los modelos. En otra parte se ha sugerido, ${ }^{15}$ y también se sugiere aquí con base en estudios llevados a cabo por el Banco Mundial sobre proyectos ya terminados a cuyo financiamiento ha contribuido, que es factible calcular para cada sector funcional de un país "coeficientes históricos de deficiencias administrativas", basados en las discrepancias medias registradas entre las estimaciones originales de los costos, beneficios y período de construcción, por una parte, y las cifras efectivas correspondientes, por la otra, de dichos proyectos. Estos coeficientes pueden utilizarse para modificar las estimaciones de los costos y el período de construcción de proyectos futuros. Sobre la base de estas estimaciones modificadas, pueden rectificarse también las relaciones capital/producto que suelen formularse partiendo de datos incorrectos o bien calcularse las relaciones capital/producto agregadas del proyecto para el sector público o para la economía en su conjunto. Es probable que estas relaciones sean mucho más correctas que las frecuentemente incluidas en los modelos de planificación.

La experiencia sugiere que también es posible medir y cuantificar el alcance de la voluntad política para alcanzar el desarrollo. Esto podría lograrse mediante: 1) la enumeración de todas las alternativas factibles en materia de inversión y de política al alcance de los dirigentes políticos; 2) el cálculo, respecto a cada alternativa, del aumento 
en los ingresos o en el producto que cabría esperar de su adopción; y 3 ) la suma de los aumentos calculados en los ingresos o el producto a que darían lugar las alternativas escogidas por los políticos encargados de la adopción de decisiones; de esta forma, se obtendría una cuantificación bastante exacta de la "voluntad política para alcanzar el desarrollo" del gobierno.

\section{Mejoramiento del proceso de planificación}

Si se incorporasen variables administrativas y políticas en los modelos de planificación, las metas de los planes serían más realistas de. lo que suelen ser.

Pero el problema de obtener mejores resultados de la planificación del desarrollo no se limita al mejoramiento de los modelos u otros aspectos de la formulación de planes a nivel macroeconómico. Si un gobierno no está dispuesto o en condiciones de tomar las medidas necesarias para la ejecución de un plan a plazo medio, dentro del contexto de la planificación convencional es imposible obtener mejores resultados de la planificación macroeconómica a plazo medio mediante la utilización de mejores modelos. Por lo tanto, los planificadores tienen que tratar de sacar el mejor partido de una situación determinada. En consecuencia, si hay razones para considerar que un país se encuentra en esta posición, sería recomendable utilizar otro enfoque a la planificación que tome esto en cuenta.

En el período de tres años y medio, durante el cual, bajo los auspicios del Banco Mundial, proporcioné asesoramiento a dieciséis países sobre sus procedimientos de planificación, tuve la oportunidad de elaborar y poner a prueba un enfoque basado en una combinación de planeamiento anual con programación sectorial, que tiene perspectivas de contribuir a que las actividades de planificación de los países de bajos ingresos produzcan resultados más satisfactorios. Partiendo del supuesto de que sería conveniente eliminar el desequilibrio que hasta ahora ha constituido la marcada inclinación hacia la planificación a plazo medio en detrimento de la planificación anual, la aplicación de métodos presupuestarios más eficaces, la programación sectorial y la preparación y ejecución de proyectos, propuse que en el proceso de planificación se prestase mayor atención a los tres elementos siguientes:

1. La preparación de planes anuales basados en dos elementos fundamentales: a) un inventario de la inversión pública en curso, racionalizado mediante la aplicación de criterios económicos generales y de otra índole y que esté en consonancia con los recursos financieros disponibles, y $b$ ) políticas encaminadas a estimular la inversión privada de características adecuadas.

2. El mejoramiento de la organización de los presupuestos, así como de su administración y procedimientos para a) establecer una relación entre los planes actuales con los presupuestos, $b$ ) establecer una relación entre la inversión y los presupuestos ordinarios, c) controlar los gastos, y d) proporcionar información sobre el progreso de los proyectos y programas.

3. La preparación de programas sectoriales multianuales, orienta- 
dos hacia la determinación de una reserva de proyectos viables en cada sector. En esencia, se trata de un esfuerzo por hacer mayor hincapié en la "planificación desde abajo", sin la cual la aplicación de la "desde arriba", que predomina en la mayor parte de los países de bajos ingresos que solamente tienen planes a plazo medio, ha dado resultados poco satisfactorios. El enfoque propuesto tiene por objeto, principalmente, mejorar la administración de las inversiones en curso y otras decisiones relacionadas con el desarrollo. Al mismo tiempo, mediante el establecimiento para cada sector de una perspectiva multianual y una "reserva" de proyectos a los que se pueda acudir en caso necesario, cada programa sectorial constituye un puente, que en la actualidad a menudo no existe, entre el plan a plazo medio y los proyectos preparados por los ministerios, departamentos y organismos técnicos. De esta forma, suele haber mayor probabilidad de que se lleven a cabo los planes a plazo medio. En países que no tienen planes a plazo medio, el enfoque propuesto contribuye a sentar una base sólida para establecerlos en forma provechosa.

E1 historial de algunos países desarrollados y en desarrollo que han tenido gran experiencia en materia de planificación proporciona apoyo a este enfoque. Al confrontar problemas especialmente difíciles o hallarse ante la perspectiva de cambios imprevisibles o situaciones de incertidumbre poco común, varios de estos países han recurrido a la planificación anual y han obtenido resultados satisfactorios. Como ya se ha señalado, en la URSS, Yugoslavia y la India, la planificación se ha efectuado o se está efectuando a base de planes anuales, porque los gobiernos respectivos decidieron que, debido a las circunstancias imperantes, por un tiempo no podrían preparar planes para períodos superiores a un año. Francia se encuentra en una situación semejante, pero está atendiendo su planificación a través de su presupuesto anual. México, que adoptó su primer plan (sexenal) en 1934, también viene atendiendo su planificación desde hace muchos años a través de su presupuesto anual, aunque en ocasiones el gobierno ha preparado planes a plazo medio. Tanto Yugoslavia como México han complementado la planificación anual con programas sectoriales multianuales.

\section{Un plan anual de acción}

En teoría, todo plan a plazo medio ha de ser seguido de planes anuales encaminados a permitir su aplicación eficaz. Los planes anuales, en mucha mayor medida que los de plazo medio, se concentran en proyectos específicos y en las medidas de política económica, fiscal, monetaria y de otra índole que debe un gobierno adoptar de inmediato si desea lograr la ejecución de un plan a plazo medio.

Los planes anuales también son necesarios para rectificar cálculos anacrónicos incluidos en los planes a plazo medio. Mediante la asignación de recursos para proyectos y programas específicos del sector público, los planes anuales constituyen la base para la confección de los presupuestos gubernamentales; y al detallar las medidas e instrumentos de política requeridos para estimular la inversión privada de acuerdo con los objetivos del desarrollo nacional, transforman las estrategias y políticas generales de desarrollo que suelen incluirse en 
los planes a plazo medio en un programa de acción para un año determinado.

En otra parte se han descrito los elementos constitutivos de un plan anual cuando éste se prepara dentro del marco de un sistema de planificación que funciona a base de planes de plazo largo y mediano.16 No obstante, como ya se ha señalado, son pocos los países de bajos ingresos que preparan planes anuales que realmente puedan considerarse como tales. Cuando no existen esos planes, resulta difícil establecer una relación entre un plan a plazo medio y los presupuestos anuales del gobierno. Ése es un defecto que podría tener consecuencias fatales, ya que para que sea posible ejecutar un plan a plazo medio, éste ha de estar reflejado en los presupuestos. La ausencia de planes anuales hace también que sea difícil traducir las estrategias y políticas de desarrollo generales de un plan a plazo medio en instrumentos específicos de política para el sector privado. En los casos en que exista un plan a plazo medio, éste con frecuencia resulta anacrónico o no constituye un marco viable para la inversión pública o la política económica, $\mathrm{y}$, por ende, tampoco para planes anuales eficaces.

En esas circunstancias, la experiencia demuestra que es conveniente preparar, para el primer ejercicio económico en que sea posible, un amplio plan anual que constituya un "plan anual de acción" mediante el cual se coordinen las actividades de desarrollo de los sectores público y privado. Un plan anual de acción puede contribuir en gran medida a reducir la asignación ineficiente de los escasos recursos y coadyuvar a la obtención de rendimientos rápidos mediante la concentración de los recursos disponibles en los proyectos de inversión pública más urgentes. Esto conlleva ventajas tanto desde un punto de vista político como económico.

Puesto que el presupuesto anual es el verdadero factor determinante de la inversión pública en muchos países de bajos ingresos, existen con frecuencia desequilibrios en la pauta de dicha inversión. El organismo presupuestario muchas veces hace asignaciones anuales para un proyecto sin saber cuánto se ha gastado ya en él, ni cuánto se necesita para terminarlo. Y es probable que el gobierno tenga aún menos información respecto a las actividades de inversión de las empresas públicas que utilizan fondos gubernamentales para realizar proyectos extrapresupuestarios.

Por lo tanto, como primer paso en la preparación de un plan anual de acción, suele ser conveniente preparar un inventario de todos los proyectos y programas públicos de inversión, incluso los patrocinados por entidades públicas. ${ }^{17}$ Paralelamente con la preparación del inventario, se establecen cálculos (como un segundo elemento del plan de acción) con dos o tres años de antelación, de los montos que se prevé que estarán disponibles para fines de inversión pública, es decir, la suma de los superávit presupuestarios en cuenta corriente, la afluencia neta prevista de préstamos y ayuda del exterior para inversiones públicas, y los préstamos internos netos obtenidos por el sector público. Es conveniente tener estos estimados cuando se prepare el plan anual

I6 Waterston, op. cit., pp. 138-142.

17 El inventario debe abarcar tanto los proyectos cuya construcción todavía no se haya iniciado como los que ya estén en marcha. 
de acción, porque los compromisos de iniciar o continuar proyectos incluidos en el plan implican que, si no quedan terminados en el año, se contará con fondos para su continuación o terminación en años posteriores.

Si el inventario de proyectos y programas de inversión pública revela, como sucede con frecuencia, que el volumen de las inversiones previstas es superior al que puede atenderse con los recursos que probablemente estarán disponibles, será preciso postergar o eliminar algunos proyectos o programas con arreglo al orden de prioridades establecido, mediante la aplicación de criterios generales de carácter económico, financiero, técnico y administrativo. ${ }^{18} \mathrm{El}$ plan de acción no debe limitarse a las asignaciones financieras para cada proyecto y programa, sino que ha de incluir estimaciones del progreso físico de cada proyecto y programa durante el año.

Como la disponibilidad de divisas suele constituir una limitación importante a la inversión en los países menos desarrollados, deben examinarse también los proyectos y programas de inversión pública que vayan a incluirse en el plan con miras a determinar sus necesidades en ese aspecto y cerciorarse de que sea posible atenderlas. Con este objeto, y como tercer punto del plan de acción, debería prepararse un presupuesto detallado de divisas para dos años o, preferiblemente, para tres; dicho presupuesto debe contener asignaciones de divisas para cada proyecto y programa incluido en el plan de acción. En el presupuesto de divisas también deben tomarse debidamente en cuenta las necesidades del sector privado.

En cuarto lugar, el plan anual de acción ha de comprender un programa de mejoras de carácter orgánico, encaminado, específicamente, a facilitar la ejecución del plan. Como se dispondrá de poco tiempo para adoptar y poner en práctica reformas administrativas y procesales, solamente deberá tratarse de implantar los cambios que, como mínimo, se requieran para la ejecución del plan. Por este motivo, deberán dejarse para una fecha posterior las modificaciones o las reformas fundamentales de la estructura del gobierno o de los procedimientos administrativos, todo lo cual es prudente suponer que será necesario.

Un quinto elemento del plan anual de acción es un programa de asistencia técnica elaborado expresamente para coadyuvar a la ejecución del plan. Dicho programa de asistencia técnica ha de determinar los consultores u otros técnicos necesarios para facilitar la realización de los proyectos del sector público que estén en marcha, la preparación de estudios de factibilidad y de preinversión en relación con proyectos potencialmente viables, o de estudios de inversión en relación con proyectos ya identificados como viables pero que todavía no estén en marcha y la modificación de las disposiciones orgánicas, o la adopción de otras nuevas, con miras a la ejecución del plan de acción.

El plan anual de acción ha de contener, como sexto y último elemento, un conjunto de medidas e instrumentos específicos de política económica, financiera, fiscal y monetaria, a través de los cuales eI

18 Los criterios y procedimientos a seguir en este aspecto se describen en Waterston, op. cit., pp. 93-99. 
gobierno procurará poner en práctica el plan y, especialmente, estimular la inversión privada de acuerdo con una orientación social satisfactoria. La mayor parte de los planes anuales se limitan, prácticamente, al sector público. Aun el Paquistán, cuyos procedimientos de planificación son excepcionalmente buenos, antes de 1968-69 solía preparar sus planes anuales solamente para el sector público.

Toda vez que la preparación adecuada de los seis elementos constitutivos, en especial el inventario racionalizado de las inversiones públicas, tomará probablemente todo el tiempo de que disponen los planificadores para elaborar el plan anual de acción, es aconsejable excluir el material de importancia secundaria. No es necesario, pues, que el plan esté basado en un modelo cuantitativo; tampoco es preciso que se establezcan metas en lo que se refiere al ingreso per capita o a la producción, ni que se haga una evaluación de la actuación económica del país en el año precedente.

No cabe duda alguna respecto a la conveniencia de incluir estos elementos, y quizá también otros. Pero la determinación de los elementos que habrán de incluirse en el plan de acción ha de basarse en el costo de oportunidad de los contados recursos de que se dispone para la planificación. Puede prescindirse de las metas agregadas en materia de ingresos y producción en el plan de acción, ya que no es probable que su inclusión permita obtener resultados mucho mejores. Los ingresos y la producción se determinan al final del año como realizaciones concretas, en lugar de al comienzo del año como metas por alcanzar. Las metas y otros elementos omitidos del plan de acción pueden incluirse en los planes anuales que sigan al plan de acción en años posteriores, en cuya oportunidad no habrá de dedicarse tiempo a racionalizar un inventario de las inversiones públicas. Respecto a la primera tentativa de planificación anual, es mejor tener un plan anual incompleto en el momento oportuno para influir sobre las decisiones básicas en materia de desarrollo, especialmente aquellas relacionadas con los presupuestos gubernamentales, que un plan detallado cuando sea demasiado tarde.

\section{La relación entre los planes y los presupuestos}

La existencia de la estrecha relación entre los planes y los presupuestos que es indispensable para la ejecución eficiente de aquéllos, exige un sistema de clasificación y procedimientos presupuestarios adecuados para traducir los elementos de los planes a términos presupuestarios, así como controles de los gastos y la información necesaria sobre los presupuestos que permita la preparación oportuna y correcta de informes trimestrales, semestrales y anuales del progreso del plan. Debido al corto tiempo de que se dispone para la preparación de un plan anual, la elaboración oportuna de informes fidedignos sobre el progreso de los proyectos que están en marcha reviste aún más importancia que en el caso de un plan a plazo medio. Pero en muchos países menos desarrollados, debido a los sistemas de clasificación presupuestaria resulta difícil relacionar los proyectos y programas incluidos en los planes con las asignaciones del presupuesto.

Una buena planificación exige también que los gastos ordinarios (por 
ejemplo, para la capacitación del personal que se empleará en un proyecto en construcción una vez terminado) se relacionen y se ajusten con los desembolsos de capital para la construcción del proyecto, de modo que todas las etapas de éste avancen en la forma necesaria para la oportuna terminación de cada fase. Además, en la planificación es útil poder tomar del presupuesto ordinario partidas de desarrollo que, al añadirse a las partidas de capital para ese fin, constituyan una exposición completa de los desembolsos presupuestarios para el desarrollo. Ahora bien, en muchos países es virtualmente imposible proceder en esa forma.

Como ya se señaló anteriormente, otro factor que obstaculiza la planificación eficaz, es que, por lo general, las estimaciones en materia de desarrollo se preparan en el organismo central de planificación, mientras que las correspondientes a los gastos ordinarios se preparan en la oficina de presupuestos, que suele depender del ministerio de finanzas. $Y$ es posible que ninguno de esos organismos tenga conocimiento de que el otro está preparando esas estimaciones hasta que estén terminadas. La falta de comunicación entre el organismo de planificación y la oficina de presupuestos no sólo impide la coordinación adecuada de los estimados de los desembolsos de capital y de los desembolsos ordinarios para fines de desarrollo, sino que también dificulta la determinación del efecto de los desembolsos corrientes para inversiones sobre los gastos ordinarios futuros. Esto constituye una grave deficiencia, ya que los gastos de capital casi siempre exigen un aumento del volumen de los gastos ordinarios. Si no se toma en cuenta este factor, puede haber incrementos imprevistos en los gastos ordinarios, lo que da lugar a la reducción del superávit en cuenta corriente disponible para inversiones de desarrollo. Otra deficiencia del mecanismo presupuestario de muchos países radica en que los ministerios técnicos tengan que hacer dos presentaciones presupuestarias, una al organismo central de planificación correspondiente a los desembolsos de capital, y otra a la oficina de presupuestos correspondiente a los ordinarios.

A pesar de que los problemas expuestos son graves, su solución no es demasiado difícil. El sistema de clasificación por finalidad que suele utilizarse en los presupuestos ordinarios, debe reemplazarse por un sistema de clasificación por funciones. Esto permite determinar qué partidas ordinarias o de capital corresponden a la categoría de desarrollo. Si se cuenta con la ayuda de un técnico en clasificación, los cambios necesarios pueden implantarse, por lo general, en un año más o menos.

En teoría, no debería ponerse en peligro la unidad del presupuesto, como en la práctica sucede en muchos países, con la preparación de los estimados de capital por un organismo y de los estimados ordinarios por otro. Pero cuando dos organismos participan en la preparación del presupuesto, cualquiera que aconseje que esa función se encomiende a un solo organismo confronta un delicado problema. Si bien el asesor que formule esa recomendación lo hará apoyado en sólidos principios, no es probable que pueda convencer a uno de los organismos a que renuncie a sus prerrogativas en favor del otro. En vista de la situación política y administrativa de muchos países, suele ser 
necesario, de momento, actuar de otra forma para lograr el mismo resultado.

Mediante el establecimiento de una Comisión de Coordinación Presupuestaria, es posible abordar en forma eficaz los problemas planteados por la dualidad de los presupuestos. Funcionarios de alta jerarquía de la oficina de presupuestos y el organismo central de planificación actúan como miembros permanentes de la Comisión, y un alto funcionario de cada ministerio, departamento u organismo técnico que formule propuestas presupuestarias actúa como miembro temporal de la misma en relación con la presentación de dichas propuestas. Cada entidad técnica presenta simultáneamente sus estimados de capital y ordinarios; éstos son examinados y discutidos por la Comisión, que los concilia entre sí y ajusta las estimaciones presupuestarias totales en función de los recursos financieros disponibles. El procedimiento descrito no sólo permite hacer estimaciones realistas del efecto de los desembolsos de capital sobre los gastos ordinarios, sino también que las entidades técnicas no tengan que hacer dos presentaciones presupuestarias, sino una sola.

\section{Programas y proyectos sectoriales}

EI valor de los planes y presupuestos anuales, en sí mismos, es limitado en lo que se refiere a influir sobre la implantación de cambios económicos básicos, ya que en un año no es mucho lo que se puede hacer en ese sentido. Por otra parte, la mayoría de los recursos disponibles suelen ya estar comprometidos para los proyectos y programas existentes. Los planes y presupuestos anuales carecen asimismo de la perspectiva necesaria para evaluar los efectos a largo plazo de las decisiones en materia de inversión. Sin esa perspectiva, es posible que decisiones que a corto plazo parezcan ser convenientes, a la larga lleguen a producir graves atascamientos y desequilibrios internos o externos.

No obstante, si se utilizan los planes y presupuestos anuales en combinación con programas sectoriales multianuales, puede contarse con cierto margen de acción y con la perspectiva necesaria. Por ejemplo, algunos proyectos incluidos en el plan anual de acción, pueden, con el tiempo, incorporarse a los programas sectoriales. Además, las consecuencias de llevar adelante proyectos en un sector básico, como la agricultura o la industria, pueden relacionarse con otros sectores, por ejemplo, mediante la terminación de proyectos complementarios de transportes y energía eléctrica. De esta manera, el plan anual de acción permite determinar ciertas inversiones "desde abajo" en cada sector. Asimismo, mediante un sistema de "regeneración" (feedback), los proyectos comprendidos en los programas sectoriales determinan qué proyectos habrán de incluirse en los planes anuales que sigan al plan anual de acción.

Sin embargo, como ya se ha apuntado, debido a la inestabilidad política, la incertidumbre económica, la adopción formalista de los planes y las deficiencias administrativas, en la mayor parte de los países de bajos ingresos resulta difícil, y en algunos imposible, la preparación de proyecciones a largo plazo y aun a plazo medio. Si los plani- 
ficadores no han tomado en cuenta la probabilidad de que, como consecuencia de los acontecimientos, sea necesario implantar cambios importantes en la orientación o alcance de su plan, la expectativa de vida del mismo podría ser breve. Pero si se han preparado para hacer frente a posibles contingencias y han previsto otras alternativas viables de desarrollo así como los proyectos y programas necesarios para su aplicación, resultará más fácil revisar el plan en caso necesario.

Los programas sectoriales pueden ser un instrumento especialmente útil para revisar los planes, si en su preparación se toma en cuenta la determinación de otras alternativas y de los proyectos necesarios para ponerlas en práctica. Por ejemplo, en el sector agrícola sería posible considerar otras alternativas para el mejoramiento e incremento de la producción de cultivos de exportación, la diversificación de las exportaciones y la sustitución de las importaciones, o combinaciones de esas actividades. Podrían determinarse los proyectos y programas requeridos en el sector agrícola para cada estrategia, y también en sectores de infraestructura conexos, por ejemplo, los transportes, el regadío y la energía eléctrica. En relación con proyectos en diferentes regiones de un país, podrían tomarse en cuenta los factores regionales. De esa forma, sería posible separar los programas sectoriales nacionales en programas regionales. El orden de prioridad de los proyectos pociria establecerse con base en la estrategia adoptada. En un programa sectorial, podrían incorporarse diferentes maneras de poner en práctica las estrategias del desarrollo. Algunas estrategias podrían llevarse a la práctica mediante la ejecución de uno o varios proyectos, mientras que otras requerirían una serie completa de proyectos determinados por el análisis de sistemas. La gama de las estrategias de desarrollo podría extenderse desde una magnitud máxima a una mínima, decidiéndose cuál habrá de aplicarse según aumenten o disminuyan los recursos. De esta manera, el ritmo de ejecución de los proyectos y programas podría acelerarse o aminorarse de acuerdo con la disponibilidad de recursos.

Cuando la programación sectorial se basa en una serie de alternativas, y la velocidad de ejecución de la alternativa elegida se determina en función de los cambios en la disponibilidad de recursos, las metas en materia de inversión y producción tienen escaso valor. No obstante, con el objeto de facilitar un enfoque coordinado, en cada sector podría adoptarse un programa renovable para los tres primeros años. Sería conveniente contar con programas coordinados para un número mayor de años, pero no es probable que un programa de esa indole sea de utilidad en condiciones de incertidumbre e inestabilidad. Los proyectos previstos para el primer año de los programas sectoriales se incluirían en el plan anual, y en los programas sectoriales renovables de tres se tomarían en cuenta los proyectos que no quedaran terminados en ese primer año. Con objeto de cerciorarse de que se tomen debidamente en cuenta y se coordinen los proyectos complementarios, se procedería a comparar entre sí los programas trienales renovables. Solamente se harían esfuerzos mínimos por relacionar entre sí los proyectos de diversos sectores más allá del trienio en cuestión, suponiendo que no es probable que la preparación de proyecciones intersectoriales para un período mayor sea fructífera. 
Los planes anuales constituirian el principal instrumento de integración, y los programas sectoriales renovables de tres años serían los elementos de coordinación secundarios, y hasta cierto punto menos detallados. En los casos en que estuvieran vigentes planes a plazo medio o largo, esos planes determinarían la composición de los anuales y de los programas sectoriales. De no existir planes de esa índole, podrían prepararse modelos parciales y, de ser posible, que coincidieran en parte, para complementar los programas sectoriales, siempre que ello no entrañase demasiado trabajo. Por ejemplo, tal vez fuera útil, y no sería difícil, proyectar el nivel de exportaciones necesario para financiar el nivel de importaciones implícito en el nivel de inversiones previsto.

La determinación de distintas estrategias de desarrollo y de los diversos proyectos necesarios para ponerlas en práctica debería ser una finalidad primordial de la programación sectorial. Una vez realizados los estudios de viabilidad y de preinversión sobre esos proyectos, se contaría con una reserva de proyectos a la cual se podría acudir según las circunstancias lo requieran, sin una demora excesiva. Esto supone que sería necesario realizar estudios sobre más proyectos que los que probablemente se llevarían a cabo. Aunque ese procedimiento tal vez parezca entrañar gastos excesivos, es probable que, a la larga, resulte menos costoso que la escasez de proyectos viables, situación en que con frecuencia se encuentran los países de bajos ingresos. En los casos en que existe una reserva de proyectos ya estudiados para un sector, resulta posible seleccionar entre distintas alternativas. Por otra parte, la existencia de esa reserva de proyectos permite a los gobiernos concertar el financiamiento de los proyectos con la anticipación necesaria para asegurar su terminación de acuerdo con el plan cronológico establecido de antemano.

\section{Planes globates a plazo más targo}

Resulta más fácil preparar planes macroeconómicos globales en los casos en que existen programas sectoriales multianuales. Por supuesto, un quinquenio nada tiene de sacrosanto, y puesto que la mayoría de los programas sectoriales se elaborarían para plazos de diez años o más, podría prepararse un plan general para diez años en lugar de cin$\mathrm{co}$, con base en los programas sectoriales. Toda vez que es probable que las proyecciones globales para un período superior a tres años más o menos no sean fidedignas ni desde el punto de vista técnico ni desde otros, no sería conveniente dedicar más de unos meses a la preparación del plan a largo plazo.

Es posible que la preparación de un plan decenal como última etapa del proceso de planificación no resulte tan satisfactoria desde un punto de vista técnico como su preparación al principio de ese proceso, que es lo que suele hacerse en la planificación convencional. Pero ése parece ser un precio bajo a pagar por los beneficios que probablemente se obtendrán cuando los planificadores inicien sus actividades con un plan anual de acción y un mejor presupuesto. Por otra parte, al proporcionar un marco global a largo plazo basado en programas sectoriales bien preparados, es probable que ese plan a largo plazo sea más sus- 
tancioso que la mayoría de los planes de esa naturaleza preparados al comienzo del proceso de planificación convencional, sin el apoyo de programas sectoriales.

\section{NeCESIDADES DE ASISTENCIA TÉCNICA}

La experiencia ha demostrado que la asistencia técnica es el requisito principal para la viabilidad, en países cuyos gobiernos estén dispuestos a adoptarlo, del enfoque que combina la planificación anual, mejores procedimientos presupuestarios y la programación sectorial. Mucho hincapié se ha hecho sobre la escasez de asistencia técnica adecuada para la planificación macro y microeconómica, así como para la programación sectorial. No cabe duda que es difícil obtener técnicos competentes, pero tal vez la escasez no sea tan grande como algunos creen. En los casos en que se ha preparado un interesante programa de acción para el desarrollo planificado, especialmente si un organismo influyente ha ofrecido su apoyo, y en que se ha llevado a cabo una intensa campaña para obtener los servicios de técnicos competentes que colaboren en la ejecución de dicho programa, generalmente se ha podido conseguir la asistencia técnica necesaria. Aun cuando la oferta de asistencia técnica adecuada es insuficiente para atender la demanda existente, la experiencia indica que si un país tiene una verdadera voluntad de promover su desarrollo, puede obtener en el extranjero la asistencia técnica que necesite. El problema aparenta ser mayor de lo que es en realidad porque muchos técnicos languidecen en países que no utilizan sus servicios a plenitud. Si se enviaran esos técnicos a países en que hacen más falta, se aliviaria, en parte, el problema que plantea su escasez.

\section{CONCLustones}

A diario los gobiernos de los países menos desarrollados adoptan decisiones sobre inversiones y otros asuntos relacionados con el desarrollo. La forma en que se adoptan y ejecutan esas decisiones refleja In situación política, económica, social y administrativa de los respectivos países. Dadas las características del subdesarrollo, esas decisiones se adoptan a menudo sin tener debidamente en cuenta el potencial en materia de desarrollo.

Frente al proceso deficiente de adopción de decisiones que suele haber en los países de bajos ingresos, cabe considerar el hecho de que para alcanzar las metas con frecuencia previstas en los planes a plazo medio, se requiere un proceso resolutorio de alta calidad. Los planificadores están conscientes de ello, desde luego, y esperan que se implanten las mejoras necesarias en dicho proceso. Pero la distancia entre la forma en que se hacen las cosas y la forma en que deberían hacerse para alcanzar los objetivos del plan suele ser demasiado grande para poder salvarla en el período de un plan a plazo medio. Por regla general, los planificadores están más familiarizados con los aspectos macroeconómicos de la planificación, que con los microeconómicos. Por lo tanto, suelen tener un concepto mucho más claro de la dirección en que ha de moverse un país para alcanzar las metas macroeconómi- 
cas, que de la manera de lograr que se produzcan los cambios políticos, administrativos y de otra índole indispensables, por lo general, para iniciar y mantener el ritmo de ese proceso. En ocasiones los planificadores consideran que su plan es como un faro en la oscuridad, y que no es necesario señalar la ruta a seguir. Por consiguiente, rara vez indican los escollos que hay que evitar durante el camino. Muchos de los que inician el viaje lo hacen con el optimismo de los inexpertos que, al encontrar que la travesía es más difícil de lo que esperaban, renuncian a continuar hasta el fin.

Los planificadores no pueden limitarse a señalar qué es lo que debe alcanzarse, sin decir quién lo va a hacer y cómo. A menos que presten atención a los medios necesarios para alcanzar las metas macroeconómicas, hay pocos motivos para esperar que el desarrollo planificado tenga en el futuro más éxito que en el pasado. El enfoque operativo a la planificación propuesto en el presente trabajo no es especialmente original ni nuevo. Si bien la secuencia y el método de planificación propuestos difieren, en algunos aspectos, de la fase de "planificación desde abajo" del procedimiento convencional, todos los elementos constitutivos del enfoque propuesto constituyen partes reconocibles del mecanismo de planificación.

El enfoque propuesto no constituye un repudio de la planificación global, puesto que la utiliza en la formulación de los planes anuales. Lo que difiere es la etapa en que se introduce la planificación global; y este cambio parece conveniente ya que es preferible mejorar el proceso de adopción de decisiones mediante la planificación anual, que postergar la tarea de abordar ese problema (como se hace en muchos países) comenzando el proceso de planificación con planes a plazo más largo. En este sentido, el enfoque propuesto es una forma de planificación "instantánea", en contraposición con la planificación "diferida" del enfoque convencional. Desde luego, conlleva un cierto riesgo de que se olvide la necesidad de una perspectiva mayor, pero este riesgo queda reducido, en gran medida, por los programas sectoriales multianuales. Al destacar la importancia de los planes anuales, el enfoque propuesto incorpora la adopción de medidas encaminadas a la ejecución del plan, como parte integral del proceso de formulación de los planes. Aunque las autoridades políticas pueden dar razones atendibles para postergar la adopción de decisiones firmes para poner en práctica un plan a plazo medio, sin quedar de inmediato expuestas a la crítica de que no tenían realmente el propósito de ejecutarlo (como sucedió en Nigeria en relación con el Plan Nacional de Desarrollo de 1962-1968), no es fácil hacerlo en el caso de un plan anual debido al corto tiempo de que se dispone para su ejecución. Ésta es una de las razones por las cuales el enfoque propuesto se denomina, acertadamente, "un enfoque operativo de la planificación del desarrollo".

El enfoque propuesto aquí puede utilizarse total o parcialmente con la planificación convencional, pero puede aplicarse por sí solo en los casos en que un país no esté en condiciones de emprender aquéIla. Aunque compatibles, los dos enfoques son diferentes. La planificación convencional persigue la solución óptima para los problemas del desarrollo, mientras que el enfoque propuesto procura lograr el máxi- 
mo aprovechamiento de las posibilidades de desarrollo con el fin de obtener "soluciones suficientemente buenas" en relación con las circunstancias políticas, administrativas y sociales de un país. A diferencia de la planificación convencional, que, para ser eficaz, suele exigir el mejoramiento sustancial a plazo medio de la situación política, administrativa y social del país, el enfoque propuesto acepta la situación tal cual existe a corto plazo.

Al igual que la planificación convencional, un objetivo del enfoque propuesto es mejorar el proceso de adopción de decisiones en materia de desarrollo; pero mientras que aquélla procede con relación a las metas que prometen los mayores rendimientos a la larga, el segundo lo hace con relación a las que prometen los rendimientos más rápidos a corto plazo.

E1 segundo enfoque no es el mejor, siempre y cuando el primero funcione como es debido. Pero si no funciona en forma satisfactoria, $y$ eso es lo que evidentemente sucede en muchos países, el segundo enfoque sería el mejor disponible, por lo menos hasta que se mejoren las disposiciones institucionales para lograr un proceso de adopción de decisiones más eficaz.

El enfoque propuesto posee ciertas ventajas con respecto al convencional. Su virtud primordial reside en que comienza con la situación actual de cada país, y aplica las técnicas de planificación que ofrecen mayores perspectivas de contribuir a mejorar la situación, en lugar de pretender que todos los países se ajusten al mismo molde, quizá con ciertas modificaciones encaminadas a atender las diferencias individuales.

Una segunda virtud es que sugiere una manera (indudablemente existen otras) de sentar, "desde abajo", una sólida base para la planificación "desde arriba". Después de todo, es el factor microeconómico el que determina, en gran medida, la actuación macroeconómica de un país. Como puede decirse que la ejecución de un plan con frecuencia comienza antes de su formulación (en el sentido de que a menudo toma más tiempo la elaboración de los proyectos que la de los planes macroeconómicos), la preparación de una reserva de proyectos para cada sector constituye una solución al problema de la "insuficiencia de proyectos".

Aunque es evidente que una estrategia de desarrollo estable es conveniente y, en realidad, esencial para la planificación convencional, el enfoque propuesto, de por sí, no depende de que continúe siguiéndose la misma estrategia de desarrollo año tras año. Por lo tanto, la flexibilidad que brinda la programación sectorial, con diversas alternativas de desarrollo, es una tercera ventaja del enfoque propuesto. Por esta razón, puede adaptarse fácilmente a la situación de inestabilidad política e incertidumbre económica que predomina en la mayor parte de los países de bajos ingresos en la actualidad.

Una cuarta ventaja es que los elementos del enfoque son separables, por lo cual cada uno puede utilizarse independientemente de los demás, lo que le proporciona aún más flexibilidad.

Una quinta ventaja estriba en que, como introduce pocos conceptos de planificación que sean nuevos, requiere pocos cambios administrativos o estructurales en la organización de los gobiernos. 
Asimismo, infunde mayor amplitud a la función de planificación al convertir en planificadores a los encargados de preparar programas sectoriales y proyectos en los ministerios, departamentos y organismos técnicos, como también a los que participan en la elaboración de los presupuestos de los gobiernos, lo que constituye una sexta ventaja de este enfoque. Para poder llevar a cabo, con éxito, los planes de desarrollo, prácticamente todos los funcionarios relacionados con la preparación de proyectos y programas sectoriales, deben considerarse a sí mismos como planificadores, y ser reconocidos como tales por los encargados de la planificación central.

Sería prematuro predecir el éxito del enfoque expuesto en este trabajo. Ahora bien, en los casos en que se ha ensayado, los resultados han sido alentadores. En varios de los dieciséis países visitados, la aplicación parcial o total de este enfoque ha contribuido a mejorar los procedimientos de planificación y los organismos correspondientes. A pesar de ser modestos, estos progresos han sido alentadores, especialmente porque, en la mayor parte de los casos, han constituido una modificación de la tendencia descendente en el proceso de planificación. 